\title{
Study on the Optimization of Hub-and-Spoke Logistics Network regarding Traffic Congestion
}

\author{
Wei Xu (iD, JinCan Huang (iD, and YanZhao Qiu \\ College of Transportation, Shandong University of Science and Technology, Qingdao 266590, China \\ Correspondence should be addressed to Wei Xu; xuwei972@163.com
}

Received 7 May 2021; Revised 20 July 2021; Accepted 4 October 2021; Published 1 November 2021

Academic Editor: Nagendra R Velaga

Copyright ( ) 2021 Wei Xu et al. This is an open access article distributed under the Creative Commons Attribution License, which permits unrestricted use, distribution, and reproduction in any medium, provided the original work is properly cited.

\begin{abstract}
The design of the hub-and-spoke network has wide applications in the freight transportation system. This design involves the location of a group of hubs as well as the allocation between nonhub nodes and the hubs after the location. On the basis of the traditional single distribution hub-and-spoke network, the congestion flow waiting model (CFWM) and the congestion flow redistribution model (CFRM) are proposed in this paper after considering traffic waiting and traffic diversion, respectively, in the case of hub congestion. The presented models focus on the design of single distribution hub-and-spoke logistics network under traffic congestion. The objective function minimizes the total cost of the road network on the premise of ensuring the normal operation of the logistics network, which effectively balances the contradiction between the economic benefits of traffic scale and the congestion cost. Given the complexity of the problem, the congestion cost function is linearized, and the mutational particle swarm optimization (MPSO) is employed for the solution. Additionally, certain calculation experiments and sensitivity analysis of the congestion optimization model are conducted to verify the effectiveness and applicability of the constructed hub-and-spoke network and the congestion solutions. The results indicate that the optimized logistics network may effectively alleviate congestion, balance the network freight flow, and improve the stability of the hub-and-spoke network.
\end{abstract}

\section{Introduction}

With the society's progress and the economy's continuous development, China is forging ahead in logistics industry. Accordingly, the logistics system is becoming increasingly perfect with the constant structural transformation and industry reform, which has become an important growth direction of national economic development. In 2019, China had the total amount of 46.1 trillion USD in social logistics, an increase of 2.3 trillion USD or 5.3\% compared with 42.8 trillion USD in 2018; though affected by the epidemic in 2020, China's total social logistics amounted to 31.3 trillion USD from January to September, remaining the strong growth tendency [1].

The current development of logistics industry in China is increasingly dependent on e-commerce. In particular, the e-commerce enterprises represented by Taobao, Jingdong, Vipshop, Dangdang, and other large-scale e-commerce platforms have provided huge support for express delivery orders. And the number of e-commerce orders in the high incidence period of online shopping tends to grow explosively. Take the Tmall Double Eleven Shopping Festival as an example. According to statistics, the all-day turnover of this festival in 2018 reached 33.02 billion USD, and the figure increased to 41.52 billion USD in 2019 [2]. The epidemic in 2020 resulted in the recession of the physical economy, which instead promoted the development of the e-commerce industry. And the all-day turnover of Tmall Double Eleven soared to 77.07 billion USD in 2020, with an increase of $233 \%$ in two years, occupying an enormous market share of e-commerce [2].

The hub-and-spoke logistics network structure was first proposed by O'Kelly, which can effectively realize the centralized transportation of goods between hubs, to generate the scale benefits of trunk transportation and offset the cost loss caused by insufficient full load rate. This network has an efficacious reduction at construction cost of transportation network and achieves economies of scale 
compared with traditional point-to-point networks, which have been widely utilized in shipping, transportation, express delivery, communication, and other fields. However, the hub-and-spoke logistics network relies excessively on the stability of the hub. In the peak season of commodity consumption, such as "Double 11 " in China or "Black Friday" in the United States, the explosive growth of orders will correspondingly promote the surge of express cargo volume. A large number of goods are concentrated in the hub for transshipment. This intensifies and aggravates the traffic pressure of the hubs and causes the flow of goods untimely transshipment, resulting in the congestion and transport delays in the hubs. If such unexpected conditions lead to the failure of the logistics network structure, it will cause the hubs overload operation or even failure. While increasing the cost of network transportation delays, the failure of one single hub will bring about the successive failure of others, leading to large-scale paralysis of the logistics network, which will not only cause incalculable cost losses, but also reduce the customer service satisfaction in a great measure. In addition to the internal factors, the impact of external ones on the network should not be underestimated, such as earthquake, flood, and disease. Such force majeure factors lead to the destruction of the hub and the failure of handling the transport flow, creating large-scale congestion of the logistics network eventually. Taking the outbreak of COVID-19 in 2019 as an example, nearly $80 \%$ of the logistics enterprises met with obstruction because of the lockdown of cities and roads, and the logistics transportation network could not operate normally and steadily, which had brought great influence and challenge to social stability and development.

Hub-and-spoke network is different from fully connected network, as shown in Figure 1. There are key hubs in hub-and-spoke network, and all goods transported from the place of departure must be dispersed through the key hub and eventually transported to the destination. In the huband-spoke network, the hubs are fully connected to form a trunk transportation network, and the nonhub nodes are assigned to the hub to form a branch transportation network. Hub-and-spoke network can concentrate network resources to the maximum extent and realize economic benefits of scale through the trunk transportation between hubs and hubs, thus reducing the overall operating cost of the network, which has been widely used in telecommunications, shipping, logistics, and other industries.

Therefore, the paper studies the hub congestion caused by the huge cargo flow during the peak consumption season taking the logistics network as an example. The congestion cost function is introduced on the basis of the traditional single-allocation logistics network, and congestion flow waiting model and congestion flow redistribution model are constructed. These two models are solved by using the methods of cut-plane and the mutation particle swarm optimization, and the sensitivity analysis of the key parameters of the network is carried out. The purpose is to minimize the cost loss of hub-and-spoke logistics network caused by the hub congestion, while effectively improving transportation efficiency of the hub-and-spoke logistics network, providing scientific reference for the construction of the hub-and-spoke logistics network.

Congestion flow waiting model (CFWM): in reality, transit hubs are capacity-constrained, and congestion occurs when the amount of goods handled exceeds their capacity. The model here considers that when congestion occurs at a hub point, the goods at the hub point can only wait in situ processing but cannot be transshipped to other hub points for transportation, thus incurring the corresponding congestion cost. The congestion flow waiting model adds mainly the cost of congestion flow compared to the traditional model.

Congestion flow redistribution model (CFRM): since congestion cost is a power function of congested traffic, when congested traffic accumulates in large quantity, congestion cost of the hubs will increase exponentially, significantly increasing the operating cost of the logistics network. In order to comprehensively consider the impact of different congestion coping strategies on network transportation costs and hub point location, CFRM introduces an alternative hub point, where the congested traffic at the hub point can be transshipped to other hub points for transportation, which will reduce congestion costs but also increase the cost of traffic transshipment.

The rest of this paper is arranged as follows: Section 2 introduces the research status of hub-and-spoke logistics network congestion and solutions at home and abroad to summarize the achievements and shortcomings of the current research; Section 3 describes and assumes the problems studied in this paper and details the basic mathematical models used in modeling; Section 4 introduces the congestion cost function and proposes most symbols. At the same time, the optimization model is used to solve the hub-and-spoke logistics network congestion problem in detail, and the mutation particle swarm optimization algorithm is applied to analyze the proposed model. Computational experiments and parameter sensitivity analysis are performed in Section 5 . Finally, Section 6 presents the conclusions of this paper.

\section{Related Work}

2.1. Literature Review. Among the studies on hub-andspoke network, many focused on discrete hub median location, since it is more in line with the actual needs. O'Kelly [3-5] was the first to propose the hub-and-spoke network structure. He combined the hub location problem with the route optimization problem, built the median model of the single assignment hub-and-spoke network, and solved it through heuristic algorithm. Camargo and Miranda [6] solved the hub location problem of single allocation in congestion based on the research of O'Kelly. The hub-andspoke network was designed from the perspective of network operators and users. And a mixed integer programming model was established. The generalized Benders Decomposition Algorithm was designed to solve large nonlinear hub-and-spoke network models quickly. Mahmoutogullari and Kara [7] proposed a duopoly model, in which two competing companies chose the hub construction sites in 


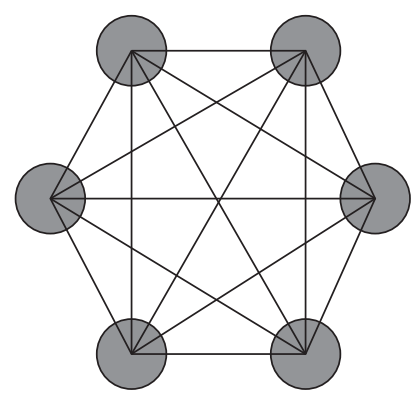

(a)

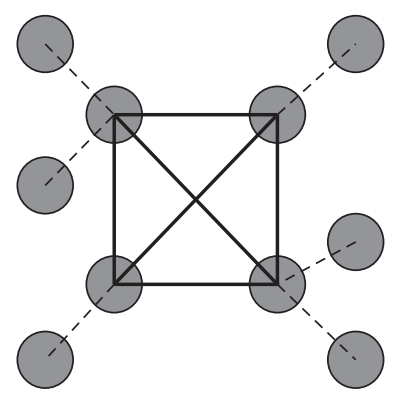

(b)

Figure 1: Comparison of hub-and-spoke network and fully connected network structure. (a) Fully connected network. (b) Hub-and-spoke network.

turn to maximize their market share under the Stackelberg game.

Many scholars have considered the uncertainty and conducted further research on the optimization problem of the hub-and-spoke logistics network. Puerto et al. [8] assumed that hub failure was accidental and its probability was known and studied the facility location of multipath transportation. Yang and Chiu [9] first considered the demand uncertainty and congestion impact and proposed a two-stage compensation model for the design of hub-andspoke network, where the hub location was decided in the first stage, and the transportation path and flow were allocated in the second one. The sensitivity analysis of model parameters was conducted through the real air transportation case, and the impact of parameter change on the solution was discussed. An et al. [10] introduced the alternative hub nodes to increase the stability of the network in the face of random failure. The location of single distribution and multidistribution hub-and-spoke logistics network was established. And the alternative bypass transportation cost was comprehensively calculated under both the normal operation and failure of hub nodes. Rahimi and TavakkoliMoghaddam [11] proposed a new dual-objective model to consider the multimodal hub location problem under the uncertainty of hub congestion and conducted sensitivity analysis on the model to deeply study the impact of congestion on the location design of hub-and-spoke network. Eghbali et al. [12] considered the hub congestion in the case of uncertainty and modeled the hub as an M/G/c queue system. An integer linear programming model was developed for the hub location problem of hub-and-spoke network with ring topology. The feasibility of this model was verified by numerous examples. $\mathrm{Wu}$ and Wang [13] constructed a modified location model considering the congestion situation of hub nodes and introduced alternative hub nodes to redistribute the congestion flow route, which promoted the balance of the flow distribution at hub nodes.

With the expansion of network scale and the increasing of network traffic, the congestion problem of hub-and-spoke logistics network is becoming more and more serious. A large number of scholars have studied the problem of quantifying and optimizing the congestion flow of hub-andspoke logistics network. Gillen and Levinson [14] proposed the calculation method of delay cost. Elhedhli and $\mathrm{Hu}$ [15] specifically described the congestion cost in the hub-andspoke network as a power exponential function through the airport flow, which is linearized by adopting the methods of line segmentation and tangent hyperplane to calculate the nonlinear congestion cost. De Camargo et al. [16] discussed the design of multidistribution star-shaped hub-and-spoke network in the case of hub congestion, modeled the congestion problem as a convex cost function, and proposed a nonlinear mixed integer programming formula. With the help of Benders Decomposition Algorithm, the standard data set problem of 81 nodes was successfully solved, which is of vital significance in effectively balancing the scale economic benefits and congestion cost loss. Camargo and Miranda [17] pointed out the problems in calculating congestion cost with the power exponential function and analyzed the cost components from the perspective of network owner and network user. Moreover, the generalized Benders Decomposition Algorithm and the external approximation method were used for model optimized solution. Kian and Kargar [18] transformed the hub location problem under congestion into two conic equations for research, which showed that when congestion cost exists, the flow in the hub-and-spoke network is no longer excessively concentrated in certain hubs and tends to be balanced instead. Fageda and Flores-Fillol [19] studied the impact of two forms of network-hub-and-spoke network and point-to-point network-on the corporate income under network congestion from the perspective of network owners. The study pointed out that the proportion of flow delay at hub nodes would increase in the case of congestion. Therefore, companies would increase the transportation frequency, generating more profit of hub-andspoke network instead, which indicated the possibility of making profit from the reasonable utilization of congestion. Azizi et al. [20] considered the stochastic demand and congestion cost based on Yang's research [9] and modeled the hub as a spatially distributed M/G/1 queue. The degree of congestion was obtained by using the expected queue length, and the impact of congestion cost on the design of hub-andspoke network was analyzed. In view of the prior reliability of hub nodes, a new decision-making framework was presented by Zhalechian et al. [21] to design a flexible hub network featuring operation and interruption risks, where the risk of damage was considered from three flexible dimensions of active capacity, response capacity, and design quality. 
Because the solution of hub-and-spoke logistics network model is a NP-Hard problem, a large number of scholars have put forward different algorithms to adapt to the changing mathematical model. $\mathrm{Hu}$ et al. [22] studied the third-party logistics system with the hub-and-spoke network of the transfer center and proposed a multiple allocation (MA) model for the design of fleet size, hub number, location, and capacity. The road congestion was modeled by transforming the route travel time formula into the function of increasing the number of trucks on the route, and a heuristic algorithm was developed to solve the nonlinear model. Alkaabneh et al. [23] comprehensively considered the scale economic benefit and hub congestion cost in the design of hub-and-spoke network and modeled the scale economic benefit as concave segment function and the congestion cost as convex function. The Lagrangian algorithm was adopted to obtain strict upper and lower limits. Effective inequalities were added to accelerate the convergence speed of the Lagrangian heuristic algorithm. The greedy stochastic adaptive search process (GRASP) was developed. The optimal design of hub-and-spoke network with scale economy and congestion cost was analyzed between nonlinear hubs, which was proven to be of vital significance to the nonlinear modeling in scale economy and congestion. Wang and $\mathrm{Wu}$ [24] established a hub location and route allocation model of single distribution hub-and-spoke logistics network (SApHLP-RC) considering failure and congestion, which can better realize the balanced distribution of logistics in the transfer center and improve the stability of the logistics network.

2.2. Results Contents. In order to clarify the research trend, the author arranges the previous section and concludes Table 1.

From the comprehensive analysis of the above studies, it can be seen that scholars mainly focus on the location problem under the constraints of unilateral actual situation at present, while less attention is paid to the overall realistic constraints, especially to the study of reliability and congestion of logistics network during the transportation concentration period. Hub failure and trunk line paralysis caused by congestion, however, will cause great economic losses. Therefore, it is crucial for transportation enterprises to study the design optimization of the hub-and-spoke network in the case of congestion situation and transfer the congestion flow in time to ensure the normal transportation of goods. Based on the traditional design of huband-spoke network and the current research gap, the congestion flow waiting model (CFWM) and the congestion flow redistribution model (CFRM) are proposed in this paper, and the influence of each parameter on the congestion situation of hub-and-spoke network is discussed in the hope of guiding the hub location, path distribution, and the transportation in the hub-and-spoke network.

\section{Problem Formulation and Hypothesis}

This chapter mainly illustrates the problems studied and puts forward the idea of optimizing the network congestion situation, on the basis of which the basic assumptions of the model are constructed, and the basic model used is introduced.

3.1. Description of the Problem Studied. It is required that each OD flow must be transferred through at least one hub node to reduce the total cost of the transportation network in the single distribution hub-and-spoke logistics network to increase the trunk flow between hubs and generate economies of scale. It is usually assumed that the trunk transportation has considerable scale benefits in the study of the hub-and-spoke network, which determines the success of the construction of the hub-and-spoke network. However, the massive concentration of trunk freight flow is bound to cause the congestion of transportation network and even the collapse of hub nodes, bringing immeasurable losses. For this reason, the congestion cost function is introduced to quantify the traffic congestion in the network. And the CFWM and CFRM are established after comprehensive consideration and analysis of the impact of economies of scale and congestion costs on the network design.

\subsection{Hypothesis of the Problem Studied}

(1) The model can only produce economies of scale through trunk transportation. The collection and distribution of freight flow will not generate scale economies, the discount of which is fixed and does not change with the flow.

(2) It is assumed that the freight transshipment can only be carried out at the confirmed hub nodes according to the characteristics of the hub-and-spoke network, and the freight transport must go through one or two hubs at most two times.

(3) The hubs in the transportation network are independent of each other. It is assumed that at most one hub node is congested at the same time to simplify the complexity of the model. Additionally, the probability of hub congestion is $p_{1}$; and the proportion of congestion flow after congestion to the total hub transportation flow is $p_{2}$.

(4) The flow within the hub's processing capacity will continue to be transferred normally and will not be affected by the congestion cost after the hub is congested. While, in the CFWM, the flow beyond the handling capacity of the hub will continue to wait for transfer at the hub, resulting in congestion waiting costs, in the CFRM, the flow beyond the hub's handling capacity will be randomly allocated to other noncongested hubs for transport, bringing about additional congestion costs and detour Cost.

3.3. Introduction to Traditional Hub-and-Spoke Network Model (THSNM). The traditional single distribution huband-spoke network model was first proposed by O'Kelly, where the number of hubs is set as $P$, and all OD flow must pass through 1 or 2 hubs for transfer. Here, the hub 
TABLE 1: Research summary.

\begin{tabular}{|c|c|c|c|c|c|c|c|}
\hline \multirow{2}{*}{ Reference } & \multirow{2}{*}{$\begin{array}{c}\text { Network } \\
\text { construction }\end{array}$} & \multicolumn{2}{|c|}{ Network optimization } & \multicolumn{2}{|c|}{$\begin{array}{c}\text { Network models and } \\
\text { algorithms }\end{array}$} & \multicolumn{2}{|c|}{ Application } \\
\hline & & $\begin{array}{c}\text { Simple } \\
\text { target }\end{array}$ & $\begin{array}{l}\text { Multiple } \\
\text { target }\end{array}$ & $\begin{array}{l}\text { Model- } \\
\text { building }\end{array}$ & $\begin{array}{c}\text { Algorithm } \\
\text { optimization }\end{array}$ & $\begin{array}{l}\text { Logistics } \\
\text { industry }\end{array}$ & $\begin{array}{l}\text { Aircraft } \\
\text { industry }\end{array}$ \\
\hline O’Kelly $[3,4]$ & $\sqrt{ }$ & $\sqrt{ }$ & & $\sqrt{ }$ & $\sqrt{ }$ & & \\
\hline $\begin{array}{l}\text { De Camargo and Miranda } \\
{[6]}\end{array}$ & $\sqrt{ }$ & & & $\sqrt{ }$ & $\sqrt{ }$ & & \\
\hline $\begin{array}{l}\text { Mahmoutogullari and Kara } \\
\text { [7] }\end{array}$ & $\sqrt{ }$ & & & $\sqrt{ }$ & & & $\sqrt{ }$ \\
\hline Puerto et al. $[8]$ & & & $\sqrt{ }$ & $\sqrt{ }$ & $\sqrt{ }$ & $\sqrt{ }$ & \\
\hline Yang and Chiu [9] & $\sqrt{ }$ & $\sqrt{ }$ & & $\sqrt{ }$ & & & $\sqrt{ }$ \\
\hline An et al. [10] & $\sqrt{ }$ & & & $\sqrt{ }$ & $\sqrt{ }$ & & \\
\hline $\begin{array}{l}\text { Rahimi and Tavakkoli- } \\
\text { Moghaddam [11] }\end{array}$ & $\sqrt{ }$ & & $\sqrt{ }$ & $\sqrt{ }$ & $\sqrt{ }$ & & \\
\hline Eghbali et al. [12] & & & $\sqrt{ }$ & $\sqrt{ }$ & $\sqrt{ }$ & & \\
\hline Wu and Wang [13] & & $\sqrt{ }$ & & $\sqrt{ }$ & $\sqrt{ }$ & $\sqrt{ }$ & \\
\hline Gillen and Levinson [14] & & $\sqrt{ }$ & & $\sqrt{ }$ & & & $\sqrt{ }$ \\
\hline Elhedhli and $\mathrm{Hu}$ [15] & $\sqrt{ }$ & & & $\sqrt{ }$ & $\sqrt{ }$ & & \\
\hline De Camargo et al. [16] & $\sqrt{ }$ & & $\sqrt{ }$ & $\sqrt{ }$ & $\sqrt{ }$ & & \\
\hline Camargo and Miranda [17] & $\sqrt{ }$ & $\sqrt{ }$ & & $\sqrt{ }$ & $\sqrt{ }$ & & \\
\hline Kian and Kargar [18] & $\sqrt{ }$ & $\sqrt{ }$ & & $\sqrt{ }$ & $\sqrt{ }$ & & \\
\hline Fageda and Flores-Fillol [19] & & & & $\sqrt{ }$ & & & $\sqrt{ }$ \\
\hline Azizi et al. [20] & $\sqrt{ }$ & $\sqrt{ }$ & & $\sqrt{ }$ & $\sqrt{ }$ & & \\
\hline Zhalechian et al. [21] & $\sqrt{ }$ & $\sqrt{ }$ & & $\sqrt{ }$ & $\sqrt{ }$ & & \\
\hline $\mathrm{Hu}$ et al. [22] & $\sqrt{ }$ & & $\sqrt{ }$ & $\sqrt{ }$ & $\sqrt{ }$ & $\sqrt{ }$ & \\
\hline Alkaabneh et al. [23] & $\sqrt{ }$ & & $\sqrt{ }$ & $\sqrt{ }$ & $\sqrt{ }$ & & \\
\hline Wang and $\mathrm{Wu}[24]$ & $\sqrt{ }$ & & $\sqrt{ }$ & $\sqrt{ }$ & $\sqrt{ }$ & $\sqrt{ }$ & \\
\hline
\end{tabular}

construction cost is included to better fit the actual operation. The model is as follows:

$$
\begin{aligned}
\min F_{1}= & \sum_{i, j, k, m} W_{i j}\left(C_{i k} Y_{i k}+\alpha C_{k m} Y_{i k} Y_{j m}+C_{j m} Y_{j m}\right) \\
& +\sum_{k} f_{k} Y_{k k} .
\end{aligned}
$$

S.t.

$$
\begin{aligned}
\sum_{k} Y_{i k} & =1 \quad \forall i \in I, \\
\sum_{k} Y_{k k} & =P, \\
Y_{i k} & \leq Y_{k k} \quad \forall i, k \in I, \\
Y_{i k} & \in\{0,1\} \quad \forall i, k \in I .
\end{aligned}
$$

Constraints (2) and (4) constrain the distribution relationship between nodes; each node can only exist as a hub node or spoke node and cannot exist independently; it can only be allocated to one hub node. Constraint (3) limits the number of hub nodes to $P$. Constraint (5) is a variable in the range of 0 to 1 .

\section{Establishment of Hub-and-Spoke Logistics Network Congestion Optimization Model}

4.1. Introduction to Congestion Cost Function. It is difficult to quantify the congestion problem of hub-and-spoke logistics network reasonably. Many studies increase the constraints of hub capacity limit to ensure that the hub points are not overutilized. It can pose a certain effect but fail to reflect the fact that hub congestion is concerned with an exponential function. Consequently, this paper introduces the comprehensive delay cost expression proposed by Elhedhli and $\mathrm{Hu}$ [15]:

$$
f(W)=\sigma W^{\tau} .
$$

This is a power exponential function, which has been widely applied in the case of single allocation. The parameter sensitivity analysis regarding congestion cost is shown in Figure 2, where $W$ is the OD flow through hub node; $\sigma$ and $\tau$ are positive constants and $\tau \geq 1$.

4.2. Description of Parameters. The notation in the paper is summarized in Table 2.

4.3. Establishment of Congestion Optimization Model. The model under traffic congestion, based on the analysis of the hub-and-spoke logistics network model, is to be broken down into three parts for explanations, including the traditional hub-and-spoke network model (THSNM), and the congestion flow waiting model (CFWM), and the congestion flow redistribution model (CFRM).

4.3.1. Congestion Flow Waiting Model (CFWM). In reality, transit hubs are capacity-constrained, and congestion occurs when the amount of goods handled exceeds their capacity. The model here considers that when congestion occurs at a hub point, the goods at the hub point can only wait in situ 


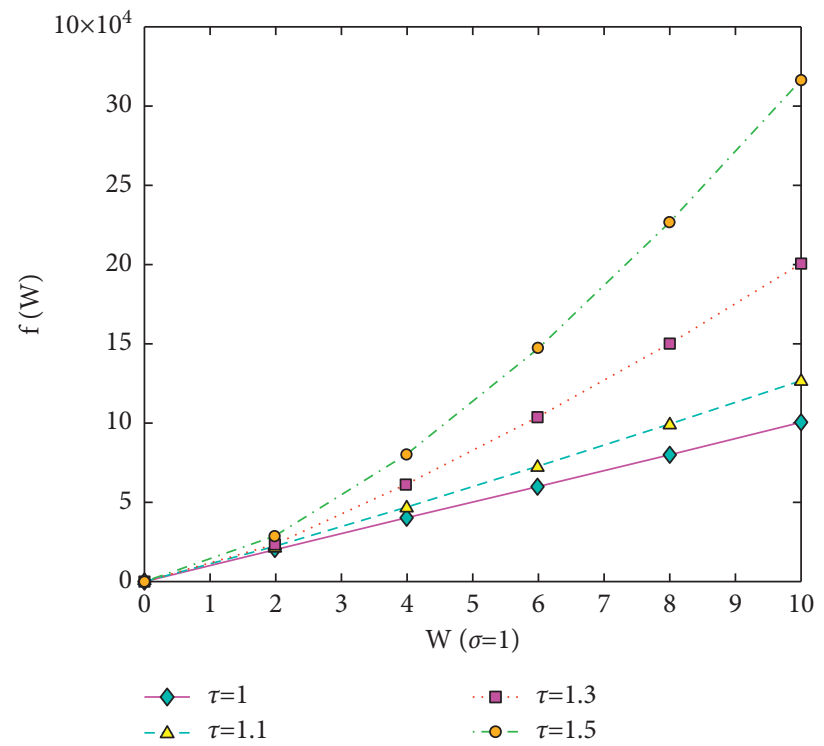

Figure 2: Influence of parameter change on congestion cost function $(\sigma=1)$.

TABle 2: Notation.

\begin{tabular}{|c|c|c|}
\hline Variable & Description & Unit \\
\hline \multicolumn{3}{|c|}{ Required data } \\
\hline$C_{i j}$ & Unit transportation cost of OD flow from node $i$ to node $j$ & $\begin{array}{l}\text { Yuan } / \mathrm{km} / \\
\text { ton }\end{array}$ \\
\hline $\begin{array}{l}D_{i j} \\
W_{i j} \\
\alpha\end{array}$ & $\begin{array}{c}\text { Transportation distance from node } i \text { to node } j \\
\text { Total OD flow from node } i \text { to node } j \\
\text { Discount factor of trunk line transportation }\end{array}$ & $\begin{array}{l}\mathrm{km} \\
\text { ton } \\
-\end{array}$ \\
\hline$C_{i k m j}$ & The transportation cost when OD flow is transported through link, $C_{i k m j}=\left(C_{i k} D_{i k}+\alpha C_{k m} D_{k m}+C_{m j} D_{m j}\right) W_{i j}$ & $\begin{array}{l}\text { Yuan } / \mathrm{km} / \\
\quad \text { ton }\end{array}$ \\
\hline $\begin{array}{l}O_{i} \\
D_{i}\end{array}$ & $\begin{array}{l}\text { Total OD flow in node } i \\
\text { Total OD flow out of node } i\end{array}$ & $\begin{array}{l}\text { ton } \\
\text { ton }\end{array}$ \\
\hline$p_{1}$ & The congestion probability of flow in the network & - \\
\hline$p_{2}$ & The proportion of congestion flow & - \\
\hline$P$ & Number of hubs & pcs \\
\hline$f_{k}$ & Hub construction cost & Yuan \\
\hline$f(W)$ & Congestion cost & Yuan \\
\hline$\sigma$ & Constant coefficient of congestion function & - \\
\hline$\tau$ & Power exponent of congestion function & - \\
\hline \multicolumn{3}{|c|}{ Decision variables } \\
\hline$X_{i k m j}$ & Binary variable. If the freight flows through $i \longrightarrow k \longrightarrow m \longrightarrow j, X_{i k m j}=1$. Otherwise, $X_{i k m j}=0$ & - \\
\hline$Y_{i k}$ & Binary variable. If node $i$ is assigned to hub $k, Z_{i k}=1$. Otherwise, $Z_{i k}=0$ & - \\
\hline$Y_{k k}$ & Binary variable. If node $k$ is selected as a hub, $Z_{k k}=1$. Otherwise, $Z_{k k}=0$ & - \\
\hline & Binary variable. If hub $k$ is congested and hub $l$ is used as a flow transfer hub, $Y_{k l}=1$. Otherwise, $Y_{k l}=0$ & - \\
\hline
\end{tabular}

processing but cannot be transshipped to other hub points for transportation, thus incurring the corresponding congestion cost.
The congestion flow waiting model adds mainly the cost of congestion flow compared to the traditional model, so the model is constructed as follows: 


$$
\begin{aligned}
\min F_{2}= & \sum_{l, k, m, i, j} W_{i j}\left(C_{i k} Y_{i k}+\alpha C_{k m} Y_{i k} Y_{j m}+C_{j m} Y_{j m}\right) Y_{l l} \\
& +\sigma\left[\left(\sum_{i} O_{i}+D_{i}\right) Y_{i l} p_{1}\right]^{\tau} p_{2}+\sum_{k} f_{k} Y_{k k} .
\end{aligned}
$$

S.t.

Constraints (2) (5) and

$$
\begin{gathered}
\sum_{j} W_{i j}=D_{i}, \\
\sum_{j} W_{i j}=O_{i}, \\
X_{i j k m}, Y_{i k}, \in\{0,1\} \quad \forall i, j, k, m ; k \neq m .
\end{gathered}
$$

The objective function is the minimalist total cost of the network. The total cost consists of three parts: a. the transportation cost of normal transportation traffic; $b$. the congestion cost of congestion at the hub point, where traffic cannot be transshipped and choose to wait for transportation, and the congestion cost is a power function about the congestion traffic; $\mathrm{c}$. the construction cost of the hub point.

Constraints (2)-(5) are the same as the traditional huband-spoke network constraints; constraints (8) and (9) calculate the overall flow out of and into node $i$; constraint (10) is a variable in the range of 0 to 1 .

In the model in this section, the OD flow passing through hubs can be expressed as $\sigma=\sum_{i} \sum_{j>i} \sum_{m} W_{i j} X_{i j k m}$; the congestion cost at hub $k$ is obtained as follows:

$$
\begin{aligned}
\sigma\left(\sum_{i} \sum_{j>i} \sum_{m} W_{i j} X_{i j k m}\right)^{\tau} & =\sigma\left(\sum_{i} \sum_{j>i} W_{i j} \sum_{m} X_{i j k m}\right)^{\tau} \\
& =\sigma\left(\sum_{i} \sum_{j>i} W_{i j} Y_{i k}\right)^{\tau} \quad \forall k .
\end{aligned}
$$

Congestion cost function is a nonlinear convex function, and great inconvenience will occur in the process of solution. It can be approximated by piecewise linearization and tangent hyperplane to turn the problem into the form of mixed linear integer [11]. We assume that the freight flow at hub $k$ is $W_{k}$, and for a given freight flow $W_{k}^{h}$, the tangent value of $f\left(W_{k}\right)$ is

$$
\begin{aligned}
f\left(W_{k}^{h}\right)+f^{\prime}\left(W_{k}^{h}\right)\left(W_{k}-W_{k}^{h}\right)= & \sigma(1-\tau)\left(W_{k}^{h}\right)^{\tau} \\
& +\mu b\left(W_{k}^{h}\right)^{\tau-1} W_{k} .
\end{aligned}
$$

Therefore, it can be linearized as follows:

$$
f\left(W_{k}\right)=\max _{h \in H_{k}}\left\{\sigma(1-\tau)\left(W_{k}^{h}\right)^{\tau}+\sigma \tau\left(W_{k}^{h}\right)^{\tau-1} W_{k}\right\},
$$

where $W_{k}^{h}, h \in H_{k}$ are a group of infinite sets that reference all points on the flow axis. Replace the congestion cost function formula in the objective function with the above results to obtain the following objective function:

$$
\begin{aligned}
\min F_{2}= & \sum_{l, k, m, i, j} W_{i j}\left(C_{i k} Y_{i k}+\alpha C_{k m} Y_{i k} Y_{j m}+C_{j m} Y_{j m}\right) Y_{l l}+\sum_{k} f_{k} Y_{k k} \\
& +\sum_{k} \max _{h \in H_{k}}\left\{\mu(1-\tau)\left(\sum_{i} \sum_{j>i} W_{i j} Z_{i k}^{h}\right)^{\tau}+\sigma \tau\left(\sum_{i} \sum_{j>i} W_{i j} Z_{i k}^{h}\right)^{\tau-1} \sum_{i} \sum_{j>i} W_{i j} Z_{i k}\right\} .
\end{aligned}
$$

Thus, there can be the following congestion flow waiting model:

$$
\begin{aligned}
\min F_{2}= & \sum_{l, k, m, i, j} W_{i j}\left(C_{i k} Y_{i k}+\alpha C_{k m} Y_{i k} Y_{j m}+C_{j m} Y_{j m}\right) Y_{l l} \\
& +\sum_{k} f_{k} Y_{k k}+\sum_{k} \Omega_{k} .
\end{aligned}
$$

The constraint conditions are (3) to (6), (8) to (10) and (16).

$$
\begin{gathered}
\Omega_{k}-\tau\left(\sum_{i} \sum_{j>i} W_{i j} Y_{i k}^{h}\right)^{\tau-1}\left(\sum_{i} \sum_{j>i} W_{i j} Y_{i k}\right) \\
\geq(1-\tau)\left(\sum_{i} \sum_{j>i} W_{i j} Y_{i k}\right)^{\tau} \quad \forall \in H_{k} .
\end{gathered}
$$


4.3.2. Congestion Flow Redistribution Model (CFRM). Since congestion cost is a power function of congested traffic, when congested traffic accumulates in large quantity, congestion cost of the hubs will increase exponentially, significantly increasing the operating cost of the logistics network. In order to comprehensively consider the impact of different congestion coping strategies on network transportation costs and hub point location, this section introduces an alternative hub point, where the congested traffic at the hub point can be transshipped to other hub points for transportation, which will not only reduce congestion costs but also increase the cost of traffic transshipment; the alternative hub transport model is constructed as follows.

$$
\begin{aligned}
\min F_{3}= & {\left[\sum_{k, l, i, m, j}\left(C_{i l m j} X_{i k m j}+C_{i l l j} X_{i k k j}\right) Z_{k l} p_{1} p_{2}+\sum_{i, j, k, m, l} C_{i k l j} X_{i k m j} Z_{m l} p_{1} p_{2}\right] } \\
& +\sum_{k} \sigma\left(\left(O_{k}+D_{k}\right) X_{k k} p_{1} p_{2}\right)^{\tau}+\sum_{i, j, k, m, l}\left[C_{i k m j} X_{i k m j} Y_{l l} p_{2}-\left(C_{i l l j} X_{i l l j}+C_{i k m j} X_{i l m j}\right) p_{1} p_{2}\right]+\sum_{k} f_{k} Y_{k k} .
\end{aligned}
$$

S.t. Constraints $(2) \sim(5)$ and

$$
\begin{gathered}
C_{i k m j}=\left(C_{i k} D_{i k}+\alpha C_{k m} D_{k m}+C_{m j} D_{m j}\right) W_{i j}, \\
Z_{k l} \leq Y_{l l}, \\
\sum_{l} Z_{k l}=Y_{k k}, \\
X_{i j k m}, Y_{i k}, Z_{k m} \in\{0,1\} \quad \forall i, j, k, m ; k \neq m . \\
\quad \sigma>0, \tau \geq 1,0 \leq p_{1} \leq 1,0 \leq p_{2} \leq 1 .
\end{gathered}
$$

The total cost consists of four parts: the first part represents the congested traffic originating from and arriving at a single hub, when the hub point is the origin and destination, which can only wait for transport but cannot be transshipped, and this traffic will incur congestion costs during transport, and this part calculates the cost of transshipping the goods through alternative hub point $l$ when the first hub point $k$ and the second hub point $m$ are congested. The second component is the detour costs incurred in reallocating congested traffic to alternative nodes in the event of congestion at a single node. The third part represents the cost of traffic transport that would normally be transported unaffected by congestion at hubs when there is hub congestion in the network. The fourth part is the construction cost of the hubs.

Constraints (2)-(5) are the same as the model above, with constraint (18) representing the transportation cost when OD flow is transported through link; and constraint (19) requires that alternative hubs can only be selected from existing hubs; and constraint (20) requires that each congested hub has only one alternative hub.

4.4. Model Solving Algorithm. Since the location and distribution of the hub-and-spoke network are a NP-hard problem, the mutational particle swarm optimization algorithm was used for the solution, and results were analyzed with the aid of MATLAB software.
Inspired by the foraging behavior of birds, MPSO was proposed by Kennedy and Eberhart in 1995. The basic principle is that a group of $m$ particles fly at a certain speed in the D-dimensional search space; when each particle searches, it takes into account the best point it has searched, as well as the best point in the history of other particles in the group, and then it changes its position on this basis. In most cases, all particles may converge faster to the optimal solution.

The standard PSO form is

$$
\begin{aligned}
& v_{i j}=w v_{i j}+c_{1} r_{1}\left(p_{i j}-x_{i j}\right)+c_{2} r_{2}\left(p_{g j}-x_{i j}\right), \\
& \begin{cases}v_{i j}=v_{\max }, \quad \text { if } v_{i j}>v_{\max } \\
v_{i j}=-v_{\max }, \quad \text { if } v_{i j}<-v_{\max }\end{cases} \\
& x_{i j}=x_{i j}+v_{i j} .
\end{aligned}
$$

In formulae (23) and (24), $c_{1}, c_{2}$ are the learning factors, $r_{1}, r_{2}$ are the pseudorandom numbers evenly distributed in the $[0,1]$ interval, $x_{i j}$ is the particle position, $v_{i j}$ is the particle speed, and $w$ is the inertia weight, whose value decreases linearly with the number of iterations.

In order to better solve the model in this paper, mutational mechanism is introduced on the basis of Standard Particle Swarm Optimization to form the Mutational Particle Swarm Optimization Algorithm, which can effectively increase the difference and nonuniformity between particles, break the balance state, and improve the efficiency of algorithm evolution.

It can be seen from equation (23) and (24) that the only driving force for its evolution is the interaction between particles, which is an internal force. When the particle swarm system with the only internal force evolves to a certain extent, the difference between particles in the particle swarm decreases, the system gradually balances, and the evolution slows down or even stagnates. This affects its ability to explore the optimal solution. In order to make the PSO algorithm have a better ability to continuously develop the optimal solution, this paper introduces the mutation mechanism on the basis of the standard particle swarm and forms the mutation particle swarm algorithm. It can 
effectively increase the differences and nonuniformity between particles, break the equilibrium state, and thus enhance the particle swarm system to improve the efficiency of system evolution. The catastrophe mechanism means that when the particle experiences the global best position, the best position is preserved, and a new seed is randomly generated. That is to say, when the optimal solution is generated, a new disturbance is added, and the search space is expanded, which is more conducive to finding the global optimal solution.

The evolution of Mutational Particle Swarm Optimization Algorithm is achieved by changing the evolutionary formula of Standard Particle Swarm Optimization Algorithm to

$$
\begin{aligned}
& v_{i j}=w v_{i j}+c_{1} r_{1}\left(p_{i j}-x_{i j}\right)+c_{2} r_{2}\left(p p-x_{i j}\right), \\
& \left\{\begin{array}{ll}
v_{i j}=v_{\max }, & \text { if } v_{i j}>v_{\max }, \\
v_{i j}=-v_{\max }, & \text { if } v_{i j}<-v_{\max }
\end{array}\right. \text {, }
\end{aligned}
$$

In formula (26), $p p$ is used to save the global optimal position that particles experienced.

The process of Mutational Particle Swarm Optimization is shown in Algorithm 1.

In order to obtain the optimal solution, the mutation particle swarm optimization algorithm can effectively avoid the possibility of traditional particle swarm algorithm falling into the local solution. The fixed number of iterations prevents the calculation from repeated execution endlessly and sets the initial value to generate the original population. Each iteration will obtain the latest solution, and the optimal solution is finally obtained by $\mathrm{N}$ times of comparison iteration.

\section{Model Simulation and Parameter Sensitivity Analysis}

5.1. Introduction to Case Basic Data. The specific case is used to analyze and verify the accuracy and reliability of the congestion optimization model proposed in this paper-a transportation company in Shandong Province. This company is mainly responsible for most of the express transportation business in Shandong Province but often experiences congestion of transportation network during the peak period. The data of transportation nodes in Shandong Province were processed after field investigation and simplified into a freight network composed of 20 nodes. The node distribution and flow demand between nodes are shown in Table 3 and Figure 3, respectively.

\subsection{Simulation Parameter-Settings}

(1) A large number of hub-and-spoke logistics network congestion cases have been studied. Based on the existing research, set the probability of traffic congestion in each hub as $p_{1}=0.2$, and the proportion of congested traffic to the total traffic as $p_{2}=0.2$;
(2) The value of discount parameter of scale economy is generally taken as $\alpha=0.7$ according to the relevant literature [5]. Two different discount parameters of scale economy are set to $\alpha=(0.2,0.7)$ to analyze the influence of scale economy on the results of the optimization model.

(3) The selection of hub nodes follows the formula $p=\sqrt{n}$ learned from the relevant literature [6]. Here, three different numbers of hub nodes were set as $p=(3,4,5)$ to analyze the impact of the number of hub nodes on the design of hub-and-spoke network under congestion.

(4) The congestion function is a power exponent function of the flow at hub nodes, highly sensitive to the change of parameters. Three groups of control experiments are set up for sensitivity analysis, with the parameter values of congestion functions taken as $\sigma=(0.1,1.5,10), \tau=(1,1.3,2)$ to analyze the impact of the parameters change in different congestion functions on the design of transportation network.

(5) There is no scale economic benefit in the collection and distribution of goods according to the related literature [5]. Therefore, the freight of goods collection and distribution at nodes were set the same with no special discount. The freight rate is set as 1 Yuan $/ \mathrm{km} /$ ton without considering the impact of the freight of goods on the network.

5.3. Model Simulation Solution. Figure 4 shows the solution results of the TM, CFWM, and CFRM when $P=4, \alpha=0.7, \sigma=1.5, \tau=1.5$. It can be found that when the hub nodes are $2,4,5$, and 8 , the number of hub-and-spoke nodes varies greatly, but the overall flow is relatively in equilibrium in the node allocation of TM through comparison. When the selected hub nodes in CFWM are 2, 4, 6, and 17 , the flow is more evenly distributed in each hub due to the heavy congestion levels at the core hubs.

In CFRM, as the flow congestion cost and the redistribution cost were taken into account, hub nodes were transferred to $2,4,6$, and 17 . It is found that the allocation of hub nodes and spoke nodes does not follow the principle of proximity but redistributed for the overall optimization.

The analysis of the data in Table 4 reveals that the flow through the hubs in CFWM tends to equalize after the introduction of congestion costs compared with the Traditional hub-and-spoke network model (TM) [5], nevertheless, leading to an increase in the total transportation cost of the network because of the rise in congestion costs. The increased total transportation cost in CFRM appears after the introduction of congestion costs and transshipment costs. Meanwhile, the hubs are classified into two types. In the case of hub congestion, nearly $70 \%$ of the traffic in the network is still transported by $50 \%$ of the hubs, while the total traffic through the hubs decreases significantly. It is pointed out that when the conflict develops between the congestion costs and economies of scale, the network will sacrifice part of the economy of scale of the 
Step 1: initialization-setting the values of $v_{\max }, c_{1}, c_{2}, w$ and the maximum number of iterations $N$. Generate the original population and calculate the fitness of individuals in the population $f$, record the $p_{i}$ and $p_{g}, p p$, keep the value of $p_{g}$, and meanwhile randomly generate the corresponding new individuals that obtain $p_{g}$.

Step 2: If the number of iterations is equal to $N$, turn to Step 5; otherwise, turn to Step 3.

Step 3: The population evolves according to formulae (26) and (25); calculate the individual fitness $f_{g}$.

Step 4: compare $f_{g}$ and $f$. If $f_{g}<f$, modify $p_{i}$ to generate a new $p_{g}$. If the value of $p_{g}$ is better than $p p$, use $p p$ to save $p_{g}$; meanwhile randomly generate the corresponding individuals that obtain $p_{g}$. Otherwise, turn to step 2.

Step 5: output the optimal individuals.

Algorithm 1: Mutational particle swarm optimization.

TABLE 3: Freight flow between nodes.

\begin{tabular}{lcccccccccccc}
\hline$D$ & 1 & 2 & 3 & 4 & 5 & 6 & 7 & 8 & 9 & 10 & $\ldots$ \\
\hline 1 & 0 & 324 & 1056 & 1062 & 730 & 523 & 861 & 436 & 295 & 809 & $\ldots$ \\
2 & 324 & 0 & 732 & 748 & 420 & 213 & 575 & 237 & 543 & 581 & $\ldots$ & 590 \\
3 & 1056 & 732 & 0 & 233 & 372 & 548 & 429 & 721 & 1237 & 615 & $\ldots$ & 969 \\
4 & 1062 & 748 & 233 & 0 & 498 & 612 & 266 & 808 & 1286 & 797 & $\ldots$ & 829 \\
5 & 730 & 420 & 372 & 0 & 0 & 208 & 492 & 353 & 877 & 327 & $\ldots$ & 855 \\
6 & 523 & 213 & 548 & 612 & 208 & 0 & 509 & 200 & 690 & 407 & $\ldots$ & 719 \\
7 & 861 & 575 & 429 & 266 & 492 & 509 & 0 & 705 & 1115 & 819 & $\ldots$ & 563 \\
8 & 436 & 237 & 721 & 808 & 353 & 200 & 705 & 0 & 528 & 376 & $\ldots$ & 825 \\
9 & 295 & 543 & 1237 & 1286 & 877 & 690 & 1115 & 528 & 0 & 844 & $\ldots$ & 954 \\
10 & 809 & 581 & 615 & 797 & 327 & 407 & 819 & 376 & 844 & 0 & $\ldots$ & 1126 \\
$\ldots$ & $\ldots$ & $\ldots$ & $\ldots$ & $\ldots$ & $\ldots$ & $\ldots$ & $\ldots$ & $\ldots$ & $\ldots$ & $\ldots$ & $\ldots$ & $\ldots$ \\
20 & 66 & 590 & 969 & 829 & 855 & 719 & 563 & 825 & 954 & 1126 & $\ldots$ & 0 \\
\hline
\end{tabular}

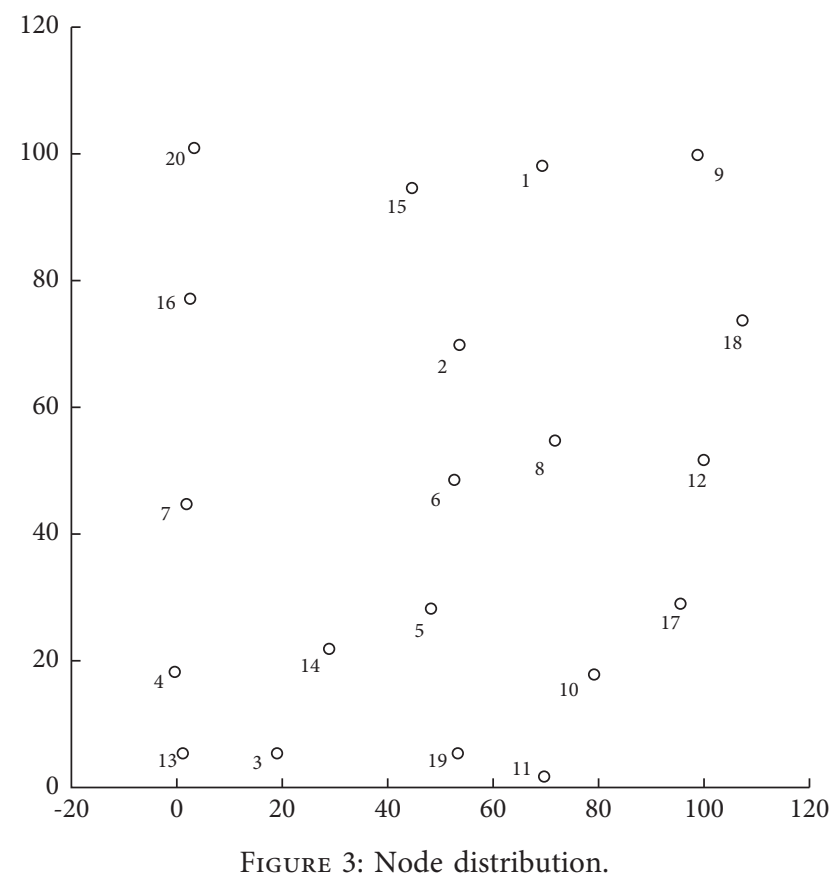

hub traffic to achieve the overall optimal network transport benefits.

5.4. Parameter Sensitivity Analysis. To effectively analyze the impact of the hub number $\mathrm{p}$ on the optimization of the flow congestion model, under the conditions when $\alpha=0.7, \sigma=1.5, \tau=1.5$, the model optimization results when $P=(3,4,5)$ were chosen, respectively, for comparison to analyze the flow imbalance ratio and the change of various costs in the network. See Table 5 for the analysis results.

To effectively analyze the impact of scale economy discount coefficient $\alpha$ of trunk line transportation between hubs on model optimization, under the conditions when $P=4, \sigma=1.5, \tau=1.5$, the model optimization results when $\alpha=(0.2,0.7)$ were picked out, respectively, for comparison, as shown in Table 6.

Finally, to effectively analyze the impact of the hub number $\sigma$ and $\tau$ on the model optimization results, under the conditions when $P=4, \alpha=0.7, \tau=1.5$, the model optimization results when $\sigma=(0.1,1.5,10)$ and $\tau=(1,1.3,1.5)$ were selected, respectively, for comparison. See Tables 7 and 8 for the analysis results.

The following conclusions can be reached based on the above experimental data.

(1) The impact of the hub number on the results of congestion model: the increase of the number of preset hubs nodes will significantly reduce the flow imbalance ratio of network hub nodes on the premise of the model unchanged, since more hub nodes mean more trunk transportation channels and more diversified node allocation modes. Although the rise in the number of hub nodes will bring the increasing construction cost under certain conditions, the transportation cost of the network can be reduced significantly in the long run. More node allocation methods may meet the requirements of node transportation distance and time to the greatest 

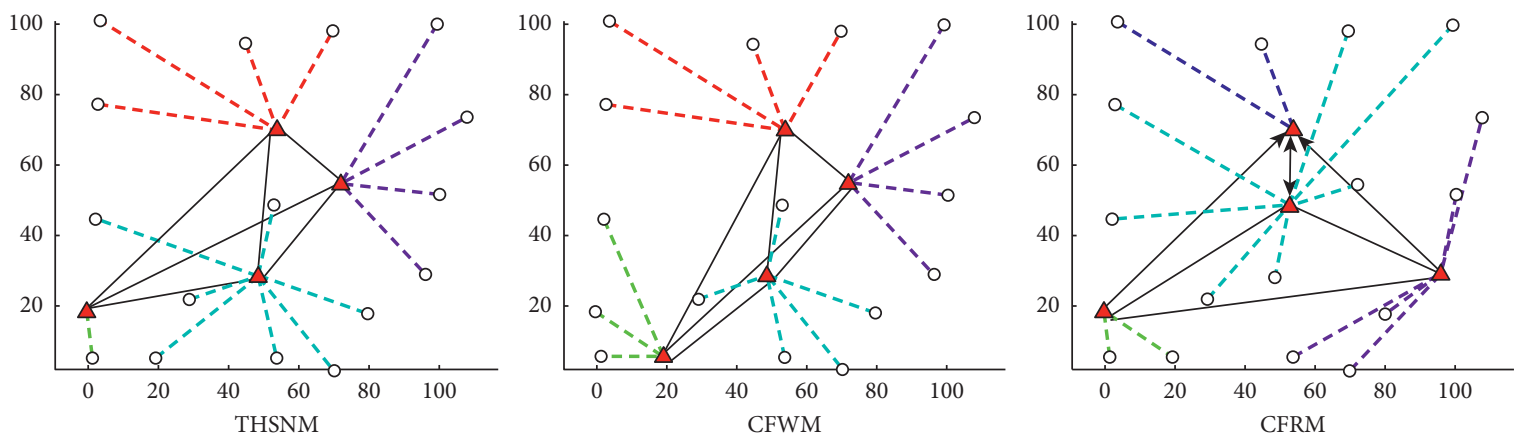

Figure 4: Comparison of model solution results.

TABLE 4: Node allocation and flow analysis.

\begin{tabular}{|c|c|c|c|c|c|}
\hline Model & Hub nodes & Flow & Freight flow imbalance ratio (\%) & Total flow through hub & Total cost \\
\hline \multirow{4}{*}{ TM } & 2 & 7755.0 & 31.58 & \multirow{4}{*}{24553.0} & \multirow{4}{*}{16811307.7} \\
\hline & 4 & 5192.0 & 21.15 & & \\
\hline & 5 & 4435.0 & 18.06 & & \\
\hline & 8 & 7171.0 & 29.21 & & \\
\hline \multirow{4}{*}{ CFWM } & 2 & 4277.0 & 24.96 & \multirow{4}{*}{17134.8} & \multirow{4}{*}{17197083.0} \\
\hline & 4 & 3543.4 & 20.68 & & \\
\hline & 6 & 4937.4 & 28.81 & & \\
\hline & 17 & 4377.0 & 25.54 & & \\
\hline \multirow{4}{*}{ CFRM } & 2 & 2801.8 & 16.79 & \multirow{4}{*}{16683.8} & \multirow{4}{*}{18008019.8} \\
\hline & 4 & 2818.0 & 16.89 & & \\
\hline & 6 & 6001.2 & 35.97 & & \\
\hline & 17 & 5062.8 & 30.35 & & \\
\hline
\end{tabular}

TABLE 5: Impact of hub number $p$ on model results.

\begin{tabular}{|c|c|c|c|c|c|c|c|c|c|}
\hline \multirow{2}{*}{$P$} & \multirow{2}{*}{ Model } & \multicolumn{5}{|c|}{ Hub flow } & \multirow{2}{*}{ Hub } & \multirow{2}{*}{ Imbalance ratio } & \multirow{2}{*}{ Total cost } \\
\hline & & H1 & $\mathrm{H} 2$ & $\mathrm{H} 3$ & $\mathrm{H} 4$ & H5 & & & \\
\hline \multirow{2}{*}{3} & CFWM & 4277 & 7105.4 & 4377 & - & - & $2,5,8$ & 1.67 & 19617523 \\
\hline & CFRM & 1999 & 2740.6 & 8870.8 & - & - & $1,4,6$ & 4.44 & 21469466 \\
\hline \multirow{2}{*}{4} & CFWM & 4277 & 3543.4 & 4937.4 & 4377 & - & $2,3,5,8$ & 1.39 & 17197083 \\
\hline & CFRM & 2801.8 & 2818 & 6001.2 & 5062.8 & - & $2,4,6,17$ & 2.12 & 18008020 \\
\hline \multirow{2}{*}{5} & CFWM & 4016.6 & 2956.6 & 3826.6 & 3168.2 & 3955.6 & $2,7,8,10,14$ & 1.36 & 16277802 \\
\hline & CFRM & 2818.6 & 2863 & 3077 & 2093 & 4144.1 & $2,7,8,10,14$ & 1.98 & 12727285 \\
\hline
\end{tabular}

TABLe 6: Impact of scale benefit discount $\alpha$ on model results.

\begin{tabular}{cccccccrr}
\hline \multirow{2}{*}{$\alpha$} & \multirow{2}{*}{ Model } & H1 & H2 & H3 & H4 & Hub & Imbalance ratio & Total cost \\
& & CFWM & 5629 & 4322.2 & 4261 & 2849.6 & $2,4,10,18$ & 1.98 \\
\multirow{2}{*}{0.2} & CFRM & 2740.6 & 2840.6 & 6117.2 & 5062.8 & $2,4,6,17$ & 2.23 & 15553791.4 \\
& CFWM & 4277 & 3543.4 & 4937.4 & 4377 & $2,3,5,8$ & 1.39 & 15112779.6 \\
\hline \multirow{2}{*}{0.7} & CFRM & 2801.8 & 2818 & 6001.2 & 5062.8 & $2,4,6,17$ & 2.12 & 19617523.1 \\
& & & & & & &
\end{tabular}

extent, effectively balancing the contradiction between the scale benefits of trunk transportation and congestion cost, to satisfy customized demands for goods transportation.

(2) The impact of scale economic benefit parameter $\alpha$ on the results of congestion model: it is found from Table 6 that the total cost is increasing with the increasing of $\alpha$, which is because the increase of $\alpha$ leads to the reduction of the scale benefit of trunk lines and the increase of the congestion cost as well. It can be seen from the change of the flow imbalance ratio that the flow imbalance ratio shows a decreasing tendency with the increasing of $\alpha$, which indicates that when the scale benefit of trunk lines is not obvious, the transportation network reduces its 
TABLE 7: Impact of congestion function parameter $\sigma$ on model results.

\begin{tabular}{|c|c|c|c|c|c|c|c|c|}
\hline \multirow{2}{*}{$\sigma$} & \multirow{2}{*}{ Model } & \multicolumn{4}{|c|}{ Hub flow } & \multirow{2}{*}{ Hub } & \multirow{2}{*}{ Imbalance ratio } & \multirow{2}{*}{ Total cost } \\
\hline & & H1 & $\mathrm{H} 2$ & H3 & H4 & & & \\
\hline \multirow{2}{*}{0.1} & CFWM & 3822.6 & 4822 & 4358.6 & 4236.2 & $2,4,6,19$ & 1.26 & 19043470.5 \\
\hline & CFRM & 3622.6 & 5022 & 4358.6 & 4236.2 & $2,6,10,19$ & 1.39 & 17297439.4 \\
\hline \multirow{2}{*}{1.5} & CFWM & 4277 & 3543.4 & 4937.4 & 4377 & $2,3,5,8$ & 1.39 & 19617523.1 \\
\hline & CFRM & 2801.8 & 2818.2 & 6001.2 & 5062.8 & $2,4,6,17$ & 2.12 & 18008019.8 \\
\hline \multirow{2}{*}{10} & CFWM & 5524.2 & 3490 & 2868.2 & 5037.2 & $2,6,10,14$ & 1.93 & 19822019.6 \\
\hline & CFRM & 3852.4 & 4776.4 & 4226.3 & 3976.8 & $4,5,10,17$ & 1.24 & 18786864.9 \\
\hline
\end{tabular}

TABLE 8: Impact of congestion function parameter $\tau$ on model results.

\begin{tabular}{|c|c|c|c|c|c|c|c|c|}
\hline \multirow{2}{*}{$\tau$} & \multirow{2}{*}{ Model } & \multicolumn{4}{|c|}{ Hub flow } & \multirow{2}{*}{ Hub } & \multirow{2}{*}{ Imbalance ratio } & \multirow{2}{*}{ Total cost } \\
\hline & & $\mathrm{H} 1$ & $\mathrm{H} 2$ & H3 & $\mathrm{H} 4$ & & & \\
\hline \multirow{2}{*}{1} & CFWM & 4277 & 4303.8 & 4327.6 & 4377 & $2,5,8,14$ & 1.02 & 19482323.2 \\
\hline & CFRM & 2827.4 & 1139 & 4379.6 & 7571.4 & $2,5,17,19$ & 6.65 & 16811307.7 \\
\hline \multirow{2}{*}{1.3} & CFWM & 4919.4 & 5058 & 2093 & 4912.4 & $2,6,10,14$ & 2.42 & 19113661.4 \\
\hline & CFRM & 2042.6 & 7587 & 2782.8 & 3672.2 & $2,4,6,19$ & 3.71 & 17468154.6 \\
\hline \multirow{2}{*}{2} & CFWM & 4814.8 & 4396.2 & 2868.2 & 5037.2 & $6,8,10,14$ & 1.76 & 19043273.1 \\
\hline & CFRM & 2442.6 & 2800.6 & 6718 & 4905.8 & $2,4,6,10$ & 2.75 & 19374806.1 \\
\hline
\end{tabular}

dependence on certain important hub nodes, and the flow is more distributed to other trunk and branch lines, but the change of $\alpha$ has little impact on the location of hub nodes.

(3) The impact of congestion function parameters $\sigma, \tau$ on the results of congestion model: analysis of the results in Table 6 and Figure 5 shows that the congestion cost in the network increases as $\sigma$ grows, and the results of CFWM and CFRM display different changes. The traffic imbalance ratio presents an upward trend in CFWM with the increasing of $\sigma$, when the effect of economies of scale in the network is greater than that of congestion cost, reducing the total cost of network operation. The freight flow imbalance ratio tends to increase and then decrease as the volume increases due to the increase of transportation costs in CFWM, demonstrating that there is a balance point among congestion cost, transportation cost, and economies of scale. When $\sigma$ transits from 0.1 to 1.5 , the impact of scale in the network is higher than that of congestion cost and transportation cost. And the increase in the unbalanced traffic ratio enhances the scale effect in the network instead, resulting in a reduction in the total cost. As $\sigma$ transits from 1.5 to 10 , the proportion of congestion cost and transportation cost increases gradually, and these two kinds of costs in the network outweigh the economies of scale, causing a large increase in total costs with the same degree of traffic imbalance ratio. Meanwhile, as can be seen from Figure 6 , the change in $\sigma$ will result in the change in the selection of hub points, which is not fixed and fluctuates greatly as $\sigma$ continues to increase.

The parameter $\tau$ is the power exponent in the congestion function, and it can be seen from Table 7 and Figure 7 that as $\tau$ increases, the degree of congestion in the CFRM increases exponentially, the flow imbalance ratio decreases, a large amount of mainline traffic is spread out to CFWM feeder transport because of high congestion costs, and the total cost increases significantly as congestion and transit costs increase, and mainline economies of scale are weakened; and the flow disequilibrium ratio shows an increasing and then decreasing trend with increasing $\tau=[1,1.3,2]$, suggesting that there is also a balance between congestion costs and economies of scale between $\tau=[1,1.3,2]$. When $\tau$ transitions from 1 to 1.3 , the effect of scale efficiency in the network is higher than the effect of congestion costs, and an increase in the traffic imbalance ratio will instead increase the scale effect in the network, resulting in a decrease in the total cost, while when $\tau$ transitions from 1.3 to 2 , the proportion of congestion costs gradually increases, its effect is greater than the scale effect, and, with the same degree of traffic imbalance ratio, it will result in a large increase in the total cost. Also, it can be seen from Figure 7 that the parameter $\tau$ has an equally large influence on the choice of the hub node, which is not fixed.

As the degree of congestion increases in the network, the imbalance ratio of traffic in the network shows a downward trend through the comprehensive analysis of the changes of parameter $\sigma, \tau$, which suggests that CFWM and CFRM play a significant role in regulating the network traffic distribution. When the scale benefit of trunk transportation is not enough to offset the losses caused by congestion cost, the model will no longer blindly pursue the scale benefit. Then, traffic will be shifted from some important hubs to other hubs or nodes to balance the distribution of traffic in the whole network. The analysis makes it clear that the congestion cost function plays a key role in the allocation of freight traffic in the network, 

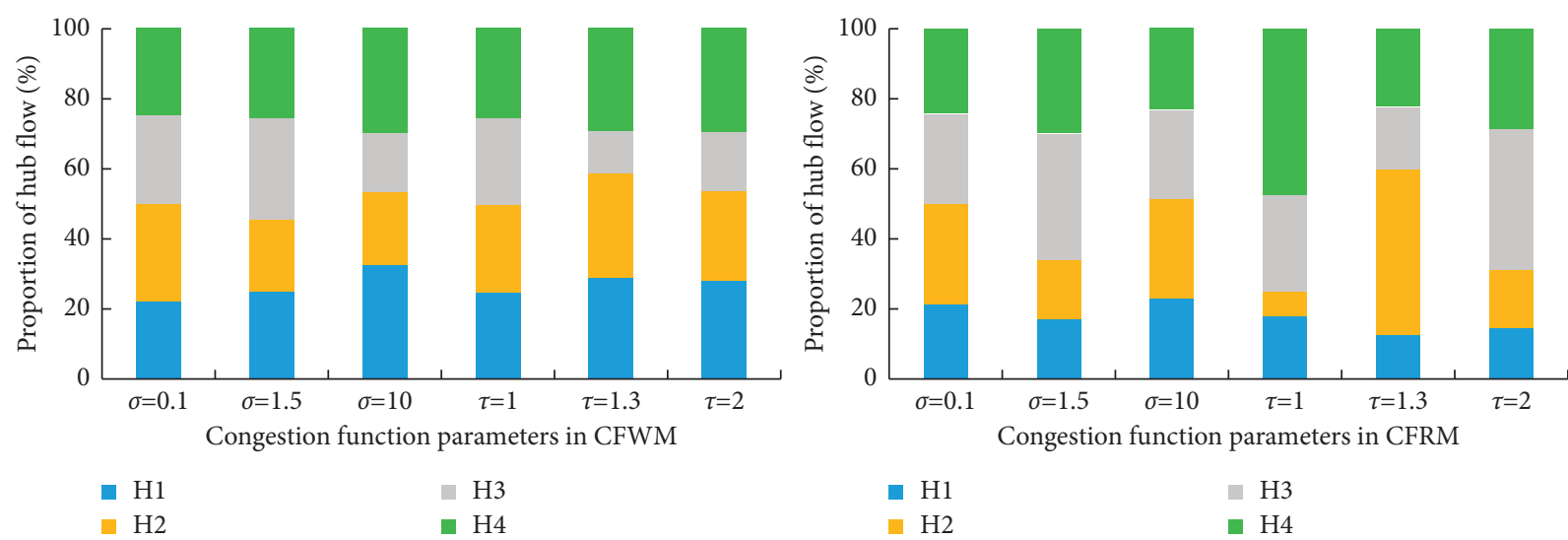

$$
\square \mathrm{H} 1
$$
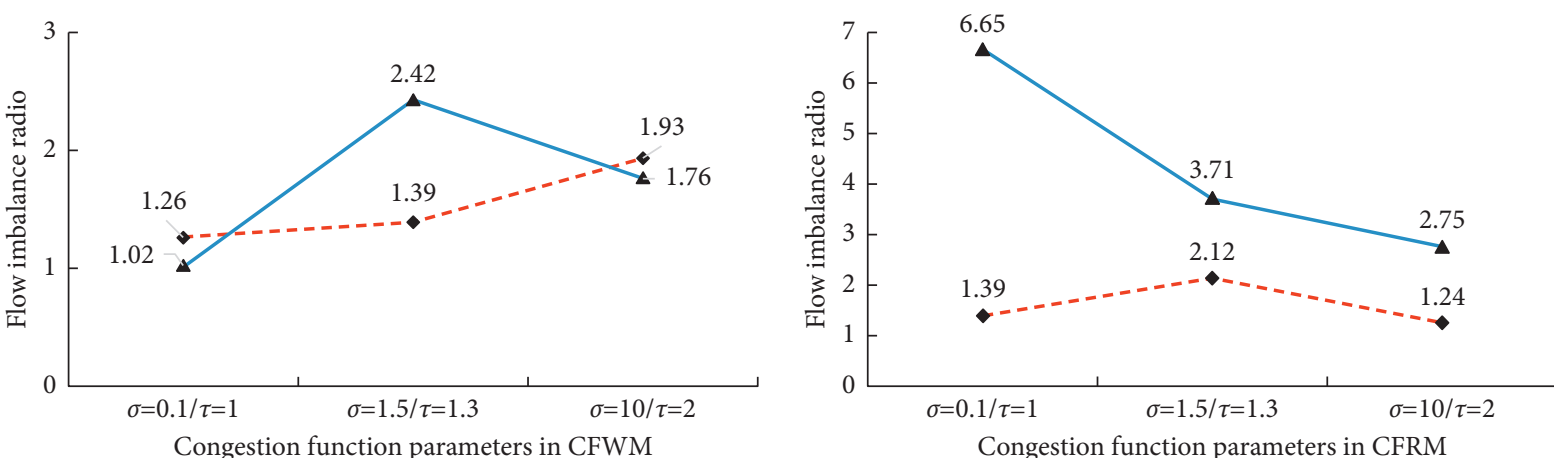

Congestion function parameters in CFWM

Congestion function parameters in CFRM

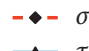

$-\bullet-\sigma$

Figure 5: Impact of congestion parameter change on flow proportion and flow imbalance ratio at each hub.
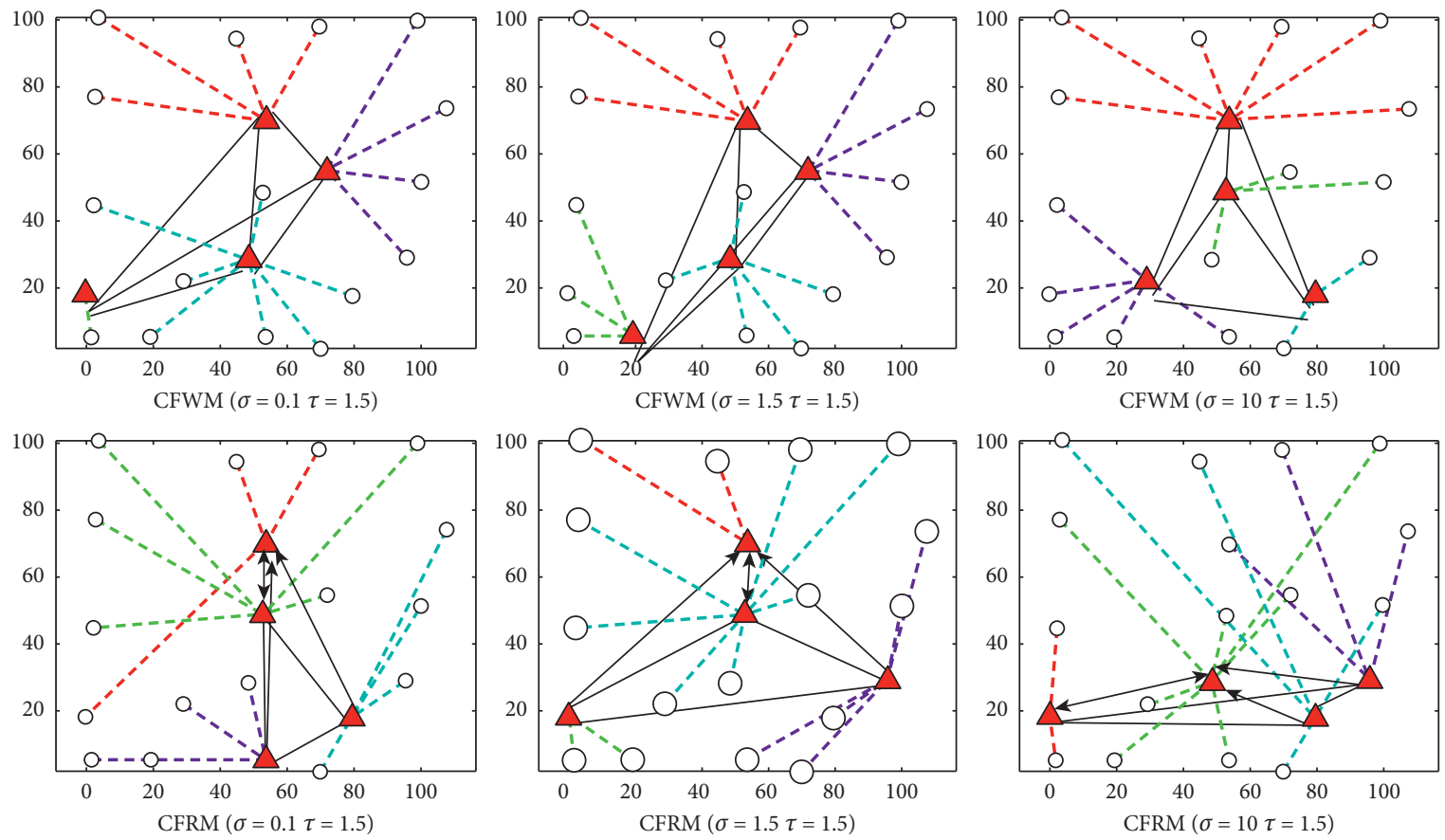

Figure 6: Influence of parameter $\sigma$ on network design. 

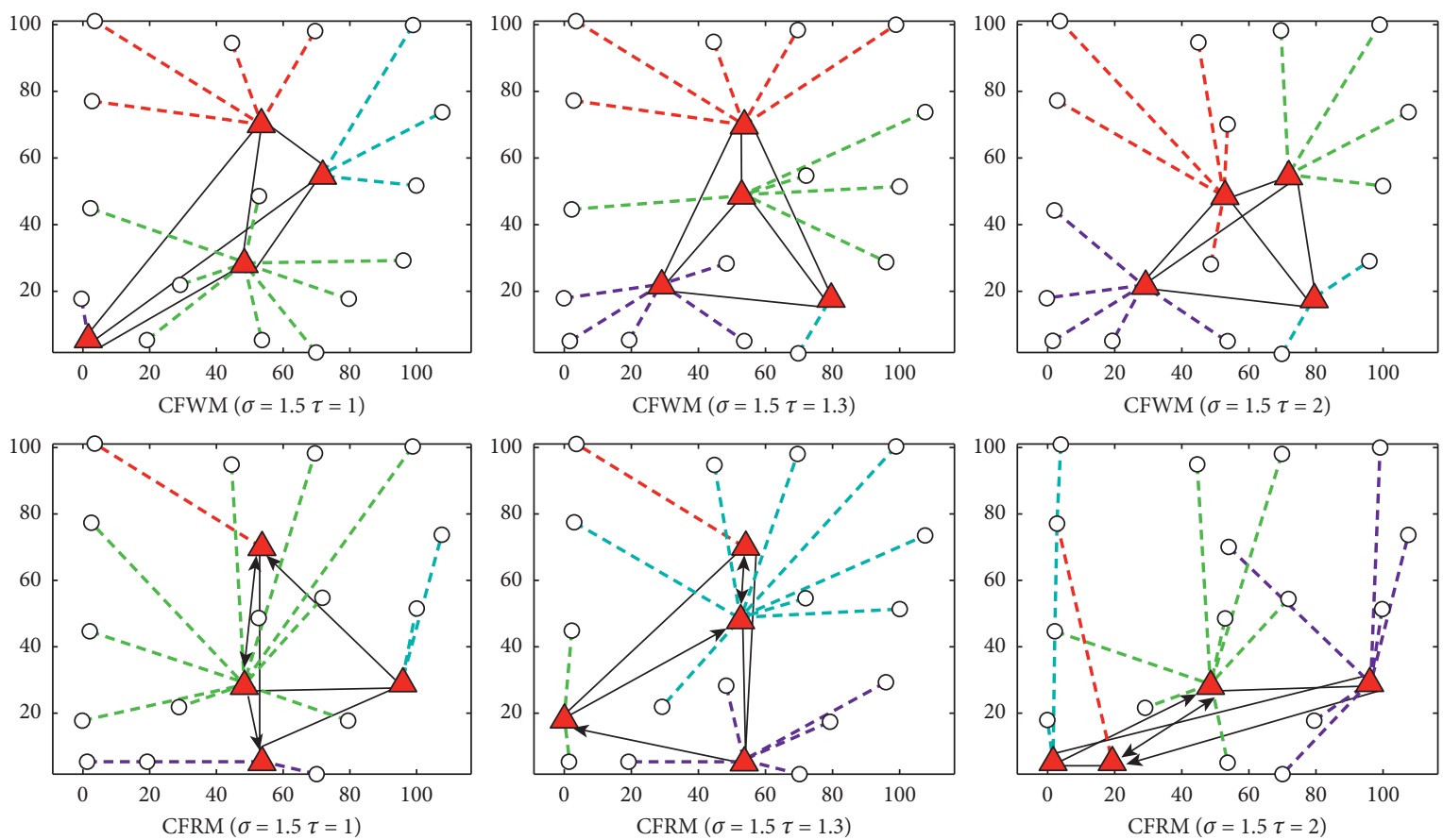

FiguRe 7: Impact of parameter $\tau$ on network design.

conducive to ease the congestion of some nodes in the network due to the excessive pursuit of economies of scale.

(4) Some nodes are frequently selected as hubs according to a group of control experiments, where the flow is relatively large, which indicates that the geographical location and inherent attributes of the nodes themselves are more important and play a decisive role, although the congestion function can change the distribution results under certain conditions.

(5) The two models (CFWM, CFRM) mentioned in this paper more fit the actual situation of the logistics operation due to increased flow congestion cost and the redistribution cost compared to the traditional model (TM). The data in Table 4 show that although part of the economy of scale of the hub traffic is sacrificed, the traffic of CFWM and CFRM is balanced under the traffic congestion scenario, avoiding massive congestion at some hub nodes and realizing the overall optimal network transportation benefit.

\section{Conclusion}

The hub-and-spoke network reduces the cost through the scale economic benefits of trunk transportation, but the following congestion will cause huge economic losses to the whole hub-and-spoke network. This paper establishes congestion flow waiting model (CFWM) and congestion flow redistribution model (CFRM) in light of the contradiction between congestion cost and scale economic benefit in a single distribution hub-and-spoke network. The linear congestion functions are introduced, and the flow transfer between hubs and the node redistribution is considered, so that the flow transportation in the network is more balanced and reasonable. The following conclusions are achieved through the comparison of model solution results. Congestion will significantly affect the overall design of the network; and the optimization model may effectively improve the freight flow equilibrium rate at hub nodes to avoid the large traffic flow concentration at some hub nodes due to the geographical advantages.

At present, the logistics network of Chinese logistics and distribution enterprises mainly has two typical structures: fully connected network and axial amplitude logistics network. For example, due to the choice of business volume of China YTO and ZTO and business positioning, they chose the axis logistics network between timeliness and cost in the trade-off for goods transportation and distribution.

Network congestion optimization, if appropriately considered by transportation enterprises, can greatly reduce the emergency cost of logistics industry while meeting the time and demand of customers. This paper reveals some interesting phenomena and suggests that:

(1) With growing congestion of hub-and-spoke logistics network, the entire transportation network needs more hubs and more hub capacity to transport the congested freight flow, which means that transportation companies have to establish new hubs to cope with the increasingly serious congestion in the transportation network. Although this will increase the cost of enterprises in a short time, this will improve the overall efficiency of logistics enterprises in the long term. 
(2) A certain balance exists among congestion cost, transshipment cost, and the scale economic benefit in the hub-and-spoke logistics network. When the congestion in the network is serious, the relationship among them conforms to the trade-off theory. Therefore, it is necessary to achieve a balance between the three under the condition of limited resources to ensure the stable operation of the transportation network. The CFWM and CFRM used in this paper provide a good way for this to adjust the network traffic distribution under congestion and find a balance among the three. Research of the models deeply improves the traditional axis spoke logistics network and provides the foundation for the future theoretical research, and the reason network layout provides a better theoretical basis for the logistics enterprises to optimize the transportation network.

(3) There are some key logistics nodes in the network, which are affected by such factors as geographical location, construction cost, and transit capacity. No matter how the network congestion status changes, these nodes are always likely to be selected as transit hubs. Therefore, enterprises should pay attention to the protection of key logistics nodes and enhance the stability of these hub nodes.

Limitations of the studied model and future research directions:

(1) The congestion flow waiting model has only one alternative hub to alleviate congestion, but the emergence of multiple alternative hubs in large complex logistics networks will make the system run more stable.

(2) The two models involved in this paper only consider one mode of transportation, but the actual transportation situation is often combined with multiple modes of transportation, such as waterway, highway, and aviation, which will increase the complexity of the model. The congestion flow waiting model and congestion flow redistribution model used in this paper are not suitable for multimodal transportation, so further optimization is needed.

(3) To simplify the research process of the hub-andspoke network, when explaining the research problem, the unit transport cost discount of the trunk transport scale economy is unchanged. The actual situation is that the trunk transport scale economy is dynamic between the unit transport cost discount coefficient and the amount of goods. When performing the example verification, to facilitate the calculation, the scale economy discount coefficient is set to a fixed value, and the transit time is not taken into account.

(4) This study on the optimization of the hub-and-spoke network only considers the network transportation of a single cargo, but in the real environment, there are many kinds of goods, and the needs of different goods for the transportation environment are inconsistent. Therefore, further research should be needed in terms of the hub-and-spoke network of many kinds of goods.

\section{Data Availability}

The (MATLAB program files) data used to support the findings of this study are available from the corresponding author upon request.

\section{Conflicts of Interest}

The authors declare that there are no conflicts of interest regarding the publication of this paper.

\section{Acknowledgments}

This research was supported in part by joint funding by Shandong Social Science Planning Research Project (18CCXJ25), Qingdao Social Science Planning Research Project (QDSKL1801134), and Qingdao Shuang Bai Research Project (2019-B-10).

\section{References}

[1] China Federation of Logistics \& Purchasing, "Analysis of logistics operation in China in 2020," China Logistics Yearbook, vol. 2, pp. 183-186, 2021.

[2] Z. L. Chen, "The marketing strategy of E-business platform-a case study of alibaba," Office Informatization, vol. 26, no. 14, pp. 26-28, 2021.

[3] M. E. O'Kelly, "The location of interacting hub facilities," Transportation Science, vol. 20, no. 2, pp. 92-106, 1986.

[4] M. E. O'Kelly, "Activity levels at hub facilities in interacting networks," Geographical Analysis, vol. 18, no. 4, pp. 343-356, 1986.

[5] M. E. O'Kelly, "A quadratic integer program for the location of interacting hub facilities," European Journal of Operational Research, vol. 32, no. 3, pp. 393-404, 1987.

[6] R. S. De Camargo and G. Miranda, "Single allocation hub location problem under congestion: network owner and user perspectives," Expert Systems with Applications, vol. 39, no. 3, pp. 3385-3391, 2012.

[7] A. I. Mahmutogullari and B. Y. Kara, "Hub location under competition," European Journal of Operational Research, vol. 250, no. 1, pp. 214-225, 2016.

[8] J. Puerto, F. Ricca, and A. Scozzari, "Reliability problems in multiple path-shaped facility location on networks," Discrete Optimization, vol. 12, pp. 61-72, 2014.

[9] T.-H. Yang and T.-Y. Chiu, "Airline hub-and-spoke system design under stochastic demand and hub congestion," Journal of Industrial and Production Engineering, vol. 33, no. 2, pp. 69-76, 2016.

[10] Y. An, Y. Zhang, and B. Zeng, "The reliable hub-and-spoke design problem: models and algorithms," Transportation Research Part B: Methodological, vol. 77, pp. 103-122, 2015.

[11] Y. Rahimi, R. Tavakkoli-Moghaddam, M. Mohammadi, and M. Sadeghi, "Multi-objective hub network design under uncertainty considering congestion: an $\mathrm{M} / \mathrm{M} / \mathrm{c} / \mathrm{K}$ queue 
system," Applied Mathematical Modelling, vol. 40, no. 5-6, pp. 4179-4198, 2016.

[12] Z. M. Eghbali, M. R. Tavakkoli, and F. Jolai, "A robustpossibilistic programming approach for a hub location problem with a ring-structured hub network under congestion: an M/G/C queue system," International Journal of Industrial Engineering, vol. 26, no. 3, pp. 273-300, 2019.

[13] Y. F. Wu and B. J. Wang, "H-S network route redistribution considering hub congestion," Systems Engineering, vol. 37, no. 3, pp. 105-114, 2019.

[14] D. Gillen and D. Levinson, "Full cost of air travel in the California corridor," Transportation Research Record: Journal of the Transportation Research Board, vol. 1662, no. 1, pp. 1-9, 1999.

[15] S. Elhedhli and F. X. Hu, "Hub-and-spoke network design with congestion," Computers \& Operations Research, vol. 32, no. 6, pp. 1615-1632, 2005.

[16] R. S. De Camargo, G. Miranda, R. P. M. Ferreira, and H. P. Luna, "Multiple allocation hub-and-spoke network design under hub congestion," Computers \& Operations Research, vol. 36, no. 12, pp. 3097-3106, 2009.

[17] R. S. De Camargo and G. D. Miranda, "Addressing congestion on single allocation hub-and-spoke networks," Pesquisa Operacional, vol. 32, no. 3, pp. 465-496, 2012.

[18] R. Kian and K. Kargar, "Comparison of the formulations for a hub-and-spoke network design problem under congestion," Computers \& Industrial Engineering, vol. 101, pp. 504-512, 2016.

[19] X. Fageda and R. Flores-Fillol, "How do airlines react to airport congestion? the role of networks," Regional Science and Urban Economics, vol. 56, pp. 73-81, 2016.

[20] N. Azizi, N. Vidyarthi, and S. S. Chauhan, "Modelling and analysis of hub-and-spoke networks under stochastic demand and congestion," Annals of Operations Research, vol. 264, no. 1-2, pp. 1-40, 2017.

[21] M. Zhalechian, S. A. Torabi, and M. Mohammadi, "Hub-andspoke network design under operational and disruption risks," Transportation Research Part E: Logistics and Transportation Review, vol. 109, pp. 20-43, 2018.

[22] L. Hu, J. X. Zhu, Y. Wang, and L. H. Lee, "Joint design of fleet size, hub locations, and hub capacities for third-party logistics networks with road congestion constraints," Transportation Research Part E: Logistics and Transportation Review, vol. 118, pp. 568-588, 2018.

[23] F. Alkaabneh, A. Diabat, and S. Elhedhli, "A Lagrangian heuristic and GRASP for the hub-and-spoke network system with economies-of-scale and congestion," Transportation Research Part C: Emerging Technologies, vol. 102, pp. 249-273, 2019.

[24] B. J. Wang and Y. F. Wu, "The hub-and-spoke network design addressing hub reliable and congestion," Industrial Engineering \& Management, vol. 24, no. 2, pp. 15-22, 2019. 